\title{
Casparian strip membrane domain proteins in Gossypium arboreum: genome-wide identification and negative regulation of lateral root growth
}

Xiaoyang Wang ${ }^{1,2}$, Yuanming Zhang ${ }^{2}$, Liyuan Wang ${ }^{1}$, Zhaoe Pan', Shoupu He1, Qiong Gao', Baojun Chen', Wenfang Gong ${ }^{1,3^{*}}$ and Xiongming Du ${ }^{1 *}$ (D)

\begin{abstract}
Background: Root systems are critical for plant growth and development. The Casparian strip in root systems is involved in stress resistance and maintaining homeostasis. Casparian strip membrane domain proteins (CASPs) are responsible for the formation of Casparian strips.

Results: To investigate the function of CASPs in cotton, we identified and characterized 48, 54, 91 and 94 CASPs from Gossypium arboreum, Gossypium raimondii, Gossypium barbadense and Gossypium hirsutum, respectively, at the genome-wide level. However, only 29 common homologous CASP genes were detected in the four Gossypium species. A collinearity analysis revealed that whole genome duplication (WGD) was the primary reason for the expansion of the genes of the CASP family in the four cotton species. However, dispersed duplication could also contribute to the expansion of the GaCASPs gene family in the ancestors of G. arboreum. Phylogenetic analysis was used to cluster a total of 85 CASP genes from G. arboreum and Arabidopsis into six distinct groups, while the genetic structure and motifs of CASPs were conserved in the same group. Most GaCASPs were expressed in diverse tissues, with the exception of that five GaCASPs (Ga08G0113, Ga08G0114, Ga08G0116, Ga08G0117 and Ga08G0118) that were highly expressed in root tissues. Analyses of the tissue and subcellular localization suggested that GaCASP27 genes (Ga08G0117) are membrane protein genes located in the root. In the GaCASP27 silenced plants and the Arabidopsis mutants, the lateral root number significantly increased. Furthermore, GaMYB36, which is related to root development was found to regulate lateral root growth by targeting GaCASP27.
\end{abstract}

Conclusions: This study provides a fundamental understanding of the CASP gene family in cotton and demonstrates the regulatory role of GaCASP27 on lateral root growth and development.

Keywords: Casparian strip membrane domain proteins (CASPS), G. arboreum, Collinearity analysis, Expression profiles, Lateral root development

\footnotetext{
*Correspondence: gwf018@126.com; dujeffrey8848@hotmail.com

'State Key Laboratory of Cotton Biology, Institute of Cotton Research, Chinese Academy of Agricultural Sciences, Anyang 455000, Henan, China

Full list of author information is available at the end of the article
}

(c) The Author(s). 2020 Open Access This article is licensed under a Creative Commons Attribution 4.0 International License, which permits use, sharing, adaptation, distribution and reproduction in any medium or format, as long as you give appropriate credit to the original author(s) and the source, provide a link to the Creative Commons licence, and indicate if changes were made. The images or other third party material in this article are included in the article's Creative Commons licence, unless indicated otherwise in a credit line to the material. If material is not included in the article's Creative Commons licence and your intended use is not permitted by statutory regulation or exceeds the permitted use, you will need to obtain permission directly from the copyright holder. To view a copy of this licence, visit http://creativecommons.org/licenses/by/4.0/ The Creative Commons Public Domain Dedication waiver (http://creativecommons.org/publicdomain/zero/1.0/) applies to the data made available in this article, unless otherwise stated in a credit line to the data. 


\section{Background}

Many higher plants have enormous and complex root systems, which are regulated by lateral roots [1]. Lateral roots are hidden below the ground, and play an important role in providing nutrients and water, which supports the rest of the plant. A large root system supports plant growth and development, and ultimately increases crop yield. For some dicotyledonous plants such as cotton, the lateral roots grow from the primary roots $[2,3]$.

Plant roots absorb nutrients from the soil through the symplast or apoplastic pathways. However, when the nutrients and water reach the root endodermis, the apoplastic pathway is blocked. To keep moving nutrients to the aerial parts, it must rely on plasma membrane special carrier proteins [4]. Blocking apoplastic flow allows the plants to adapt to various environmental changes [4]. This specialized structure forms a hydrophobic band called the "Casparian strip" [5]. When defective mutants are present in the Casparian strip, it fails to maintain ion homeostasis due to the inward or outward leakage of xylem ions, which causes abnormal phenotypes in adverse soil conditions [6]. The formation of the Casparian strips depends on Casparian strip membrane domain proteins (CASPs), which are primarily responsible for the accumulation of lignin polymerization in the central region of endodermal plasma membranes [7]. Five Casparian strip membrane domain proteins, containing four transmembrane domains were identified in Arabidopsis roots, CASP1 and CASP3 play vital roles during the formation of Casparian strips. The CASPs are located in the plasma membrane, and interact with secreted peroxidases, directly modifying the cell wall with their membrane domain $[8,9]$. Furthermore, the peroxidase $(P R X)$ genes and monolignol oxidizing enzyme genes, such as Respiratory burst oxidase homologue $F(R B O H F)$ and laccases $(L A C s)$ are preferentially accumulated in the endodermis, which are necessary for the formation of Casparian strips [10]. The plant peptide hormone, named "Casparian strip integrity factor" (CIF1/2), binds to the leucine-rich repeat receptor kinase SCHENGEN3 (SGN3) and is necessary for the contiguous formation of Casparian strips [6]. The SGN3 mutants severely disrupted the Casparian strips without altering the concentration of most ions, with the exception of magnesium and potassium homeostasis [11]. SGN1, a receptor-like cytoplasmic kinase (RLCK), ensures the Casparian strip membrane domain is in the correct position [12]. The transcription factor SHORTROOT (SHR) targets another transcription factor, MYB36, and is also responsible for the development of Casparian strips [13].

Cotton is a natural fiber from which textiles are manufactured, and also provides cottonseed oil [14]. Cotton root systems include one primary root and numerous lateral roots, while the lateral root development affects the whole root system. Larger cotton root systems can resist nutrient deficiency by efficiently absorbing $\mathrm{K}^{+}[15,16]$. However, the restricted root system reduces cotton photosynthesis and biomass production [17]. Some genes play important roles in regulating cotton lateral root development: GhARG, a cotton arginase gene, represses the formation of the lateral root. The GhARG silenced cotton plants that were grow well under both low and high nitric conditions $[18,19]$. Additionally, the GhSTOP1 gene positively affects lateral root development when exposed to acid stress [20]. Using RNAi technology to down-regulate GhSTOP1 in cotton decreases the expression of genes related to lateral root development and delays the growth of lateral roots.

Compared with allotetraploid cotton which has a complex genome (AADD), diploid Asiatic cotton (G. arboreum) has a simple genome (AA), which makes it a valuable resource for studying agricultural and morphological traits. The published genomic data of G. hirsutum, G. barbadense, G. raimondii and G. arboreum helps analyze of CASP genes at the genome-wide level in cotton. However, the specific function of the CASP gene family in Gossypium is largely unknown. The relationship between CASP proteins and root development in cotton has yet to be identified. In this study, 48 CASP genes were identified. They were randomly distributed on all 13 chromosomes of G. arboreum. Among them, 29 were identified in the four Gossypium species. Most CASP genes exhibited a high level of expression in the initial growth stages of fiber, and roots, stems and leaves in vegetable tissues. However, five GaCASP genes (Ga08G0113, Ga08G0114, Ga08G0116, Ga08G0117 and Ga08G0118) were exclusively expressed in roots. Histochemical analysis showed that GaCASP27 was particularly expressed in roots. This study outlines findings that will aid in the further identification of the functions of CASP genes in root development, which can be utilized to breed new varieties with large root systems.

\section{Results \\ Identification and chromosomal distribution analysis of CASP genes in Gossypium}

Our previous study identified four Casparian-strip membrane protein genes in 215 G. arboreum accessions [21]. These homologous genes included Ga08G0114, Ga08G0116, Ga08G0117, and Ga08G0118. This study used Ga08G0117 sequence as a query, and downloaded PF04535 from Pfam database (http://pfam.xfam.org/) using Hidden Markov Model (HMM) analysis [22]. We searched the cotton protein dataset using the HMMER3.0 software [23]. A total of 49, 57, 110, and 101 CASP genes were identified from G. arboreum (diploid), G. raimondii (diploid), G. barbadense (tetraploid), and G. hirsutum (tetraploid), respectively. The putative CASP genes were then analyzed using SMART 
(Simple Modular Architecture Research Tool) and NCBICDD databases (https://www.ncbi.nlm.nih.gov/cdd) to identify the common domain of the CASPs, using the threshold $\left(\mathrm{E}<10^{-14}\right)$. Finally, 48, 54, 91 and 94. CASP genes were identified from G. arboreum, G. raimondii, G. barbadense and G. hirsutum, respectively. The genes GaCASP1 to GaCASP48, GbCASP1 to GbCASP91, GrCASP1 to GbCASP54 and GhCASP1 to GhCASP94 were named based on their genetic IDs in the genome database. The chromosomal locations of the CASPs were collected from cotton FGD (https://cottonfgd.org/) and are listed in Additional file 2. These genes were unevenly distributed on different Gossypium chromosomes. The chromosomal distribution of GaCASP genes was constructed by the Mapchart software based on their chromosomal location (Additional file 3, Additional file 8). GaCASP genes were randomly distributed over all 13 chromosomes of $G$. arboreum. The Ga08G0114, Ga08G0116, Ga08G0117, and Ga08G0118 genes were located on the same Chr8, indicating that these genes could have been duplicated from an identical gene and perform similar functions. However, the CASP gene Ga14G0035 was not mapped on any chromosomes (Additional file 8). Furthermore, consecutively numbered genes in the same chromosome showed similar molecular weights and lengths of proteins, such as the diploid Asiatic cotton genes Ga08G0113 and Ga08G0114, Ga08G0116, Ga08G0117 and Ga08G0118, the tetraploid upland cotton genes Gh_A08G0061, Gh_A08G0062, Gh_ A08G0063, Gh_A08G0064 and Gh_A08G0065, the tetraploid island cotton genes GOBAR_DD14175, GOBAR DD14176, GOBAR_DD14177, and GOBAR_DD14178, and the diploid D genome genes Gorai.004G010700.1, Gorai.004G010800.1, Gorai.004G010900.1, Gorai.004G0110 00.1, and Gorai.004G011100.1. Among all of the identified CASP proteins, GhSca109203G01 was the smallest protein, with 69 amino acids (aa). The largest was Gh D09G1628 (325 aa). The molecular weight of the proteins ranged from 7.746 to $35.878 \mathrm{kDa}$, while the isoelectric point ranged from 3.894 (Gh_A10G1948) to 11.672(Gorai.012G052700.1). Detailed information regarding Casparian strip proteins in cotton is available in Additional file 2.

\section{Collinearity analysis of 48 Casparian strip genes from $\mathbf{G}$.} arboreum compared with G. barbadense, G. hirsutum and G. raimondii

Previous studies have demonstrated that whole-genome, tandem and segmental duplications play central roles in the expansion of the Gossypium gene family $[24,25]$. A chromosomal region within $200 \mathrm{~kb}$ containing two or more consecutive genes is defined as a tandem duplication event [26]. To reveal the genome-wide duplicated mechanism of the CASP gene family in G. arboreum, all intragenomic duplication data was filtered by MCScanX. Four tandem duplicated gene pairs (Ga03G0527.1/
Ga03G0528.1, Ga08G0113.1/Ga08G0114.1, Ga08G01 16.1/Ga08G0117.1, and Ga08G0116.1/Ga08G0117.1) were detected in G. arboreum. Nine tandem duplicated gene pairs were detected in G. hirsutum and G. barbadense, while no tandem duplicated gene pairs were detected in G. raimondii. Throughout the whole genomic analysis, 18, 44, 66 and 72 gene pairs were considered whole genome duplication (WGD) in G. arboreum, G. raimondii, G. barbadense and G. hirsutum, respectively. There are 21, 10, 10 and 10 gene pairs that are considered dispersed duplications in G. arboreum, G. raimondii, G. barbadense and G. hirsutum, respectively. Two gene pairs were detected as proximal duplications in G. arboreum. However, other gossypium did not detect proximal duplication of the CASP gene family. As a result, whole genome and dispersed duplications could be the primary driving forces for the expansion of the CASP gene family in Gossypium (Additional file 4). Tandem duplication events occurred less frequently, suggesting they it might not play a key role in the expansion of GaCASPs, GrCASPs, GbCASPs and GhCASPs. A total of 70,45 and 39 orthologous CASP gene pairs were detected between G. arboreum and G. hirsutum, G. barbadense and G. raimondii using TBtools software, respectively. We identified a total of 29 common homologous CASP gene pairs in the four Gossypium species. Details for the collinear gene pairs are listed in Fig. 1 and Additional file 5.

\section{Phylogenetic analysis and classification of CASP genes}

Members of the GaCASPs family have conserved extracellular loops as well as the standard topology of fourmembrane spans with cytosolic amino and carboxy termini (Fig. 2a), which is consistent with those found in Arabidopsis [7]. Furthermore, we randomly selected six CASP genes (Ga08G0117, Gh_A08G0064, Gh_D08 G0103, GOBAR_AA16400.1, GOBAR_DD14177.1 and Gorai.004G011000) from each clade to perform further protein sequence analyses. The CASP paralogs proteins were highly conserved in amino acids, which contained a domain that could have catalytic activity with a conserved arginine and aspartate, forming an active site (Fig. 2b). These proteins contain four transmembrane helices. In order to understand the similarities and differences in GaCASPs between cotton and Arabidopsis, the phylogenetic tree was constructed using 48 CASP protein sequences from $G$. arboreum and 37 CASP sequences from Arabidopsis. Subsequent phylogenetic analysis indicated that CASPs were mainly grouped into six separate subfamilies (Fig. 2c). Clade I had 32 members, followed by Clade IV (19), CladeII (18) and CladeIII (9). Clade V had only two members, while Clade V and VI contained only Arabidopsis genes. 


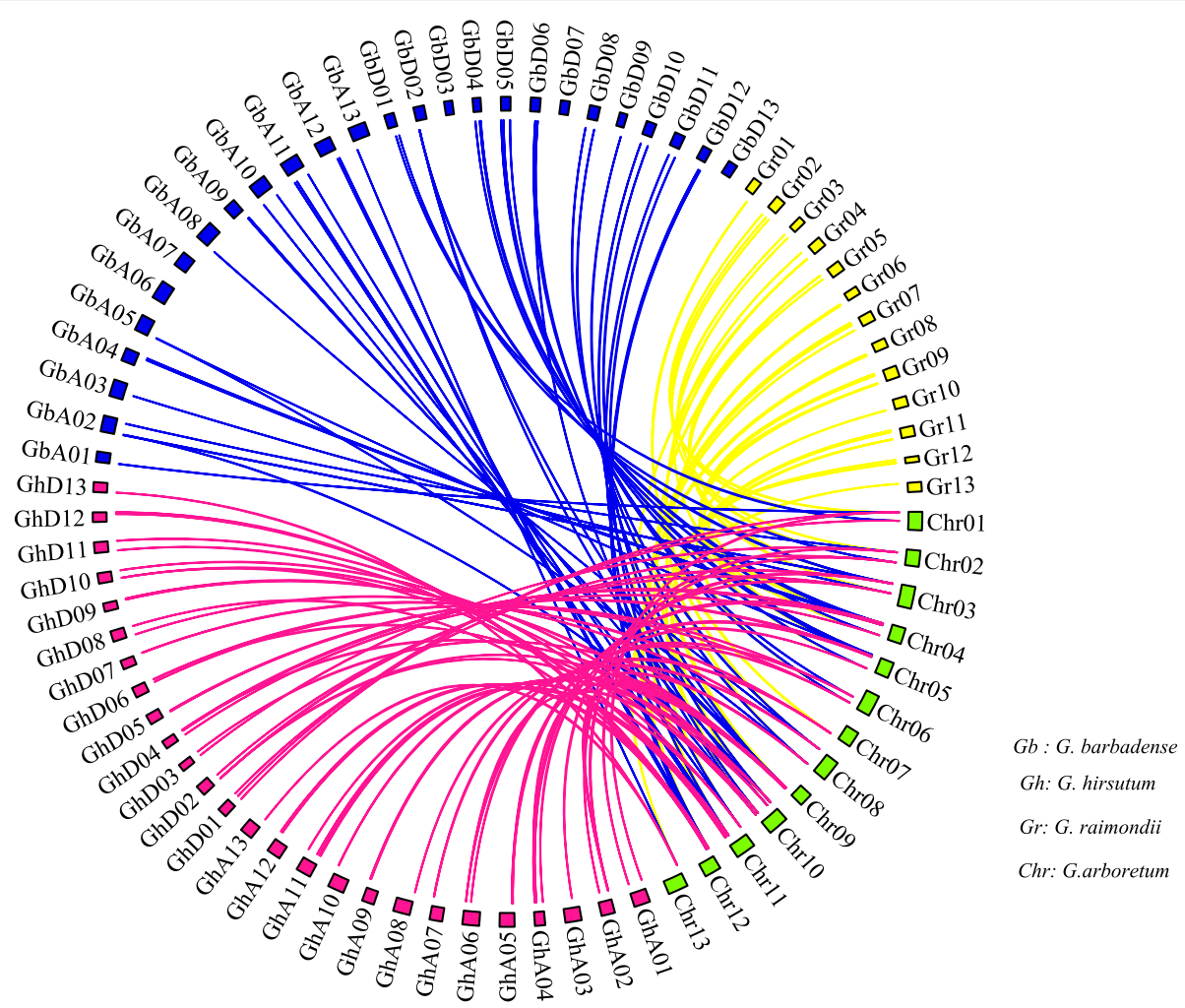

Fig. 1 Microsynteny analysis of CASP genes between G. arboretum and G. hirsutum, G. barbadense and G. raimondii. Red lines connect the homologous genes between G. arboretum and G. hirsutum, yellow lines connect the homologous genes between G. arboretum and G. raimondii, and blue lines connect the homologous genes between G. arboretum and G. barbadense. Green, pink, blue, and yellow boxes indicate the G. arboretum, G. hirsutum, G. barbadense and G. raimondii chromosomes, respectively

Gene structure and conserved motif composition of $G$. arboreum CASP gene family

To gain more insight into the evolution of the GaCASP family in G. arboreum, we examined how the exons and introns were organized in all the identified GaCASP genes and constructed a phylogenetic tree using 48 GaCASPs protein sequences. Forty-eight genes were divided into four groups, while most CASP genes typically contained three exons and two introns (Fig. 3a). Ga08G0113, Ga08G0114, Ga08G0116, and Ga08G0118 possessed a similar exon-intron structure, but the Ga08G0117 gene possessed a long exon-intron structure (Fig. 3b). Further analysis of the MEME motif was used to predict the protein-conserved motifs in GaCASPs. Ten distinct motifs were identified, while GaCASP proteins in the same group typically shared a similar motif composition (Fig. 3c). Nine motifs were identified in Clade I, with the exception of motif 4 . Clade II contained motif 8 , motif 2 , motif 5 ,and motif 7 . Clade III contained motif 1 , motif 9 , and motif 10 . Clade IV contained motif 6 , motif 3 , motif 4 , and motif 1 . Overall, the GaCASP members of the same group shared similar conserved motif compositions and gene structures.
Along with the results of our phylogenetic analysis, this strongly supports the reliability of the group classification results.

Expression profiling of G. arboreum CASP genes with RNA-seq In order to elucidate the possible role of CASPs in the root growth and development of G. arboreum, we investigated the expression patterns of 48 GaCASPS in different developmental stages of fiber, root, stem and leaf tissues using the transcriptome data, (Additional file 6 and Fig. 4). In order to further classify the gene expression patterns of the 48 GaCASPs gene, these genes were classified using hierarchical Clustering Software Cluster3.0 following their statistical analysis. The expression patterns were then divided into five major clusters, based on tree branching. Most CASP genes were expressed in all tissues, but some CASP family members were only expressed in the roots, such as the genes in group II. Only one gene, Ga01G0372 (classified into the fifth group) was significantly expressed in different stages of fiber development but showed weaker expression in roots. The genes in the first group displayed low expression levels in all of the detected tissue. The genes in the second group were 
A

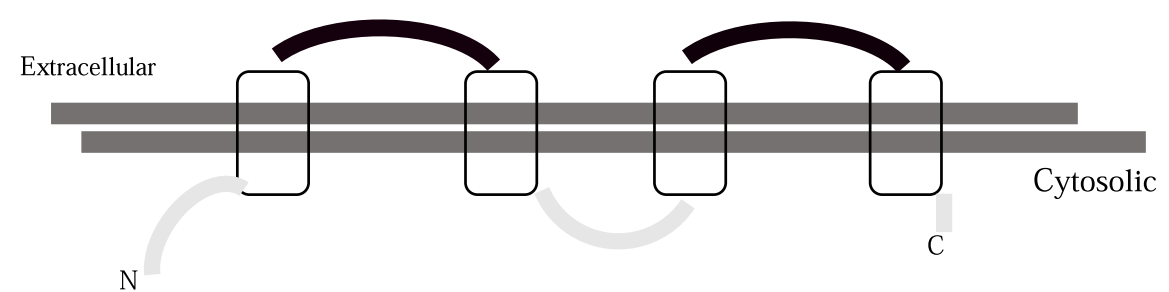

B
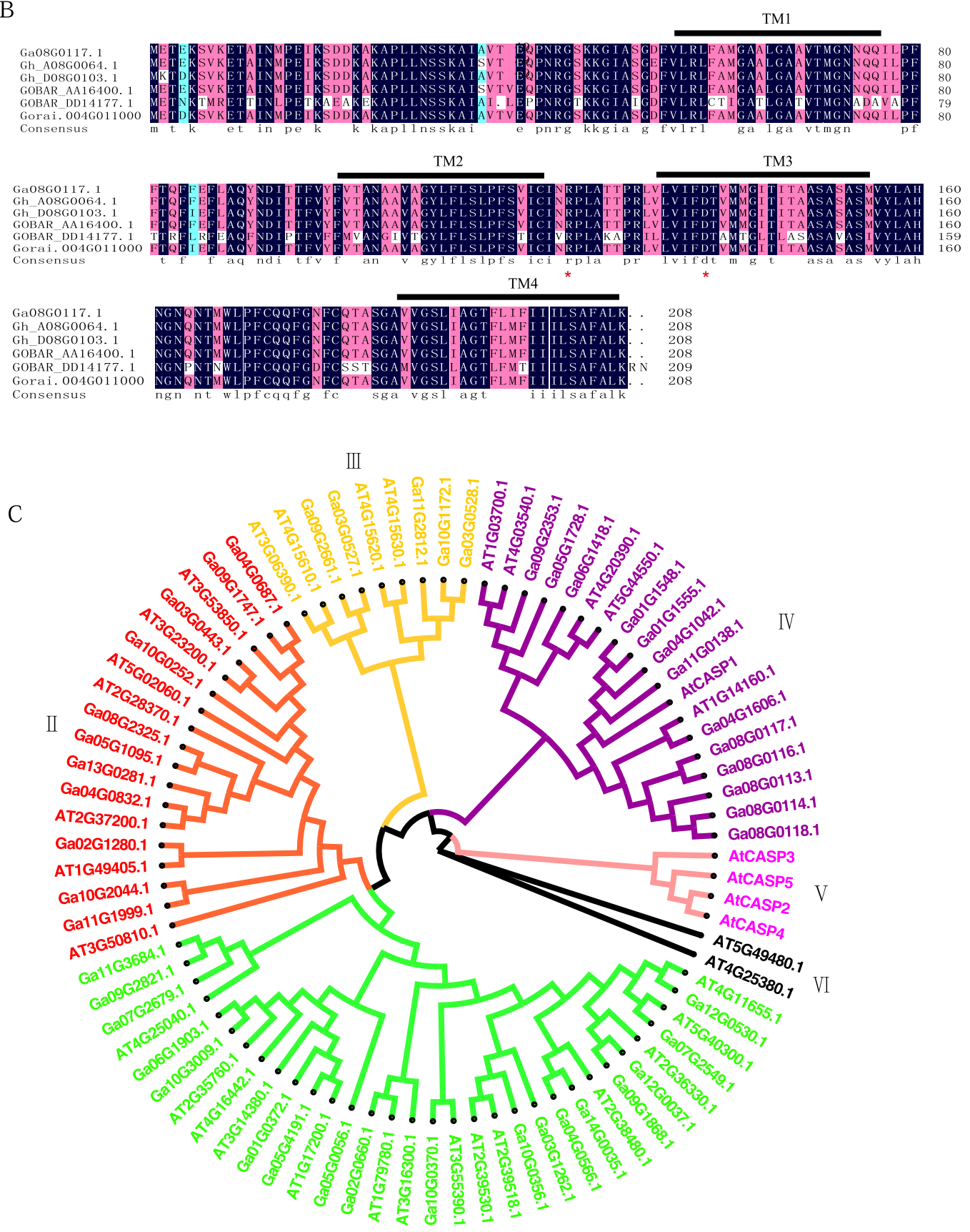

Fig. 2 (See legend on next page.) 
(See figure on previous page.)

Fig. 2 Topology, conserved Casparian strip membrane domain features, and phylogenetic tree of the CASP family. a Predicted topology of the GaCASPs, the four gray boxes represent the four-membrane spans, the arc lines indicate cytosolic amino, carboxy termini, and conserved extracellular loops. b Multiple alignment and transmembrane region analysis of GaCASP, GhCASP, GrCASP, and GbCASP protein sequences. The four transmembrane (TM) domains were analyzed using the TMHMM program. c Phylogenetic relationships of CASPs between Arabidopsis and G. arboretum. The phylogenetic tree was constructed by the MEGA 7 program based on the protein sequences. The maximum likelihood method was used and bootstrap values were carried out 1000 replications

highly expressed in roots. As shown in the yellow box, these genes included: Ga08G0113, Ga08G0114, Ga08G0116, Ga08G0117, and Ga08G0118 (red letters) as well as other CASP like genes in this family. The third group contained 13 genes that were highly expressed in all the detected tissues. The gene Ga11G2812 (in the fourth group) was expressed across the different development stages of fibers, however, no expression was detected in the vegetable tissues (stems, leaves and roots). These results indicated that certain CASPs had different spatial and temporal expression patterns in cotton.

\section{Tissue expression and subcellular localization of GaCASP3 protein}

In order to further confirm the localization of expression of CASP genes, we selected the GaCASP27 gene (Ga08G0117)

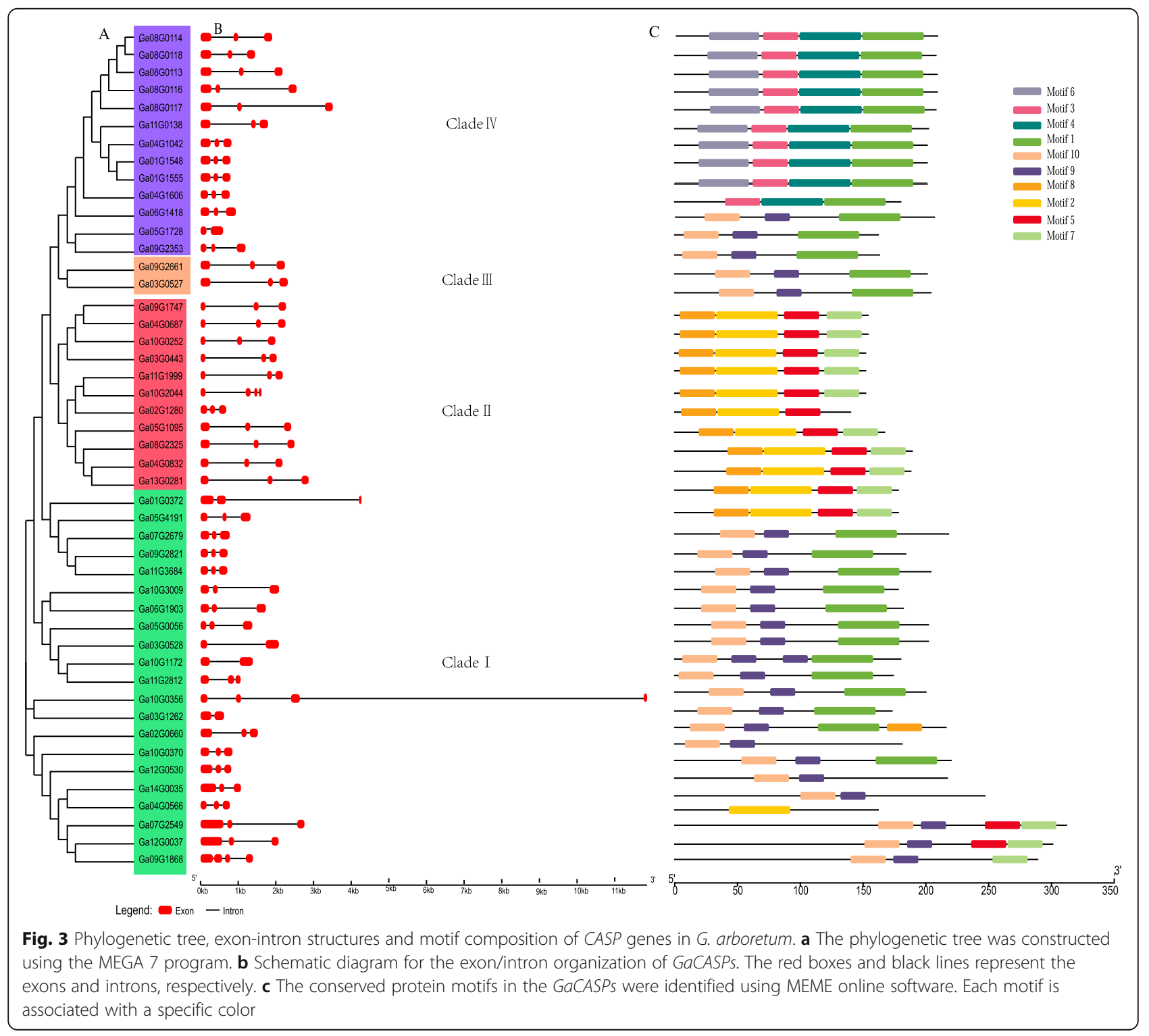




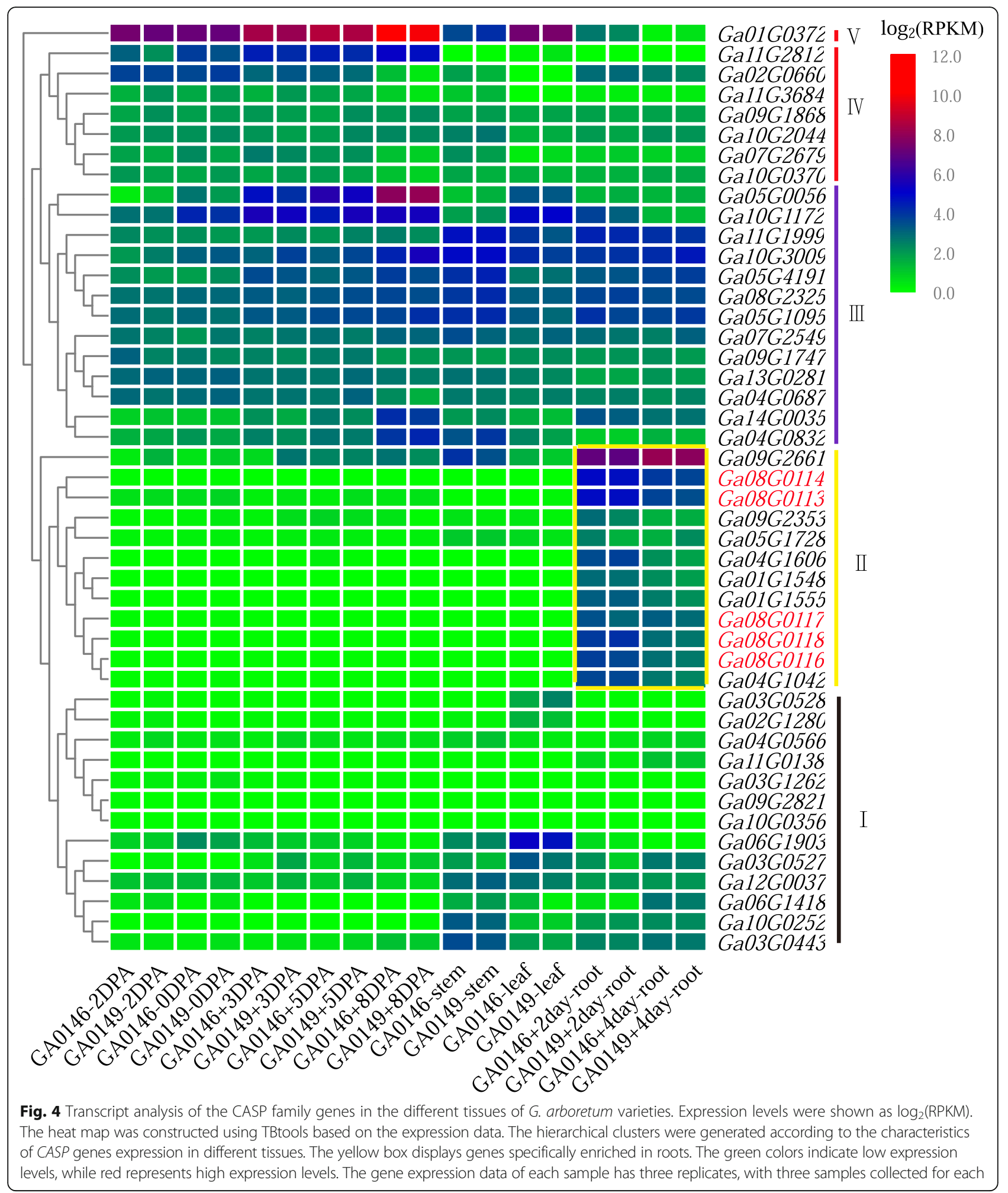

to construct a GaCASP27-promoter-GUS (for $\beta$-glucuronidase) reporter vector, followed by transformation into Arabidopsis (Additional file 9). We observed intense GUS staining in the roots (Fig. 5a), indicating that GaCASP27 was primarily expressed in roots.
According to the online tools TargetP and SignalP, the GaCASP27 protein should be localized in the plasma membrane. Subcellular localization of the GaCASP27 protein was determined by the construction of a GaCASP27-green fluorescent protein (GFP) fusion 

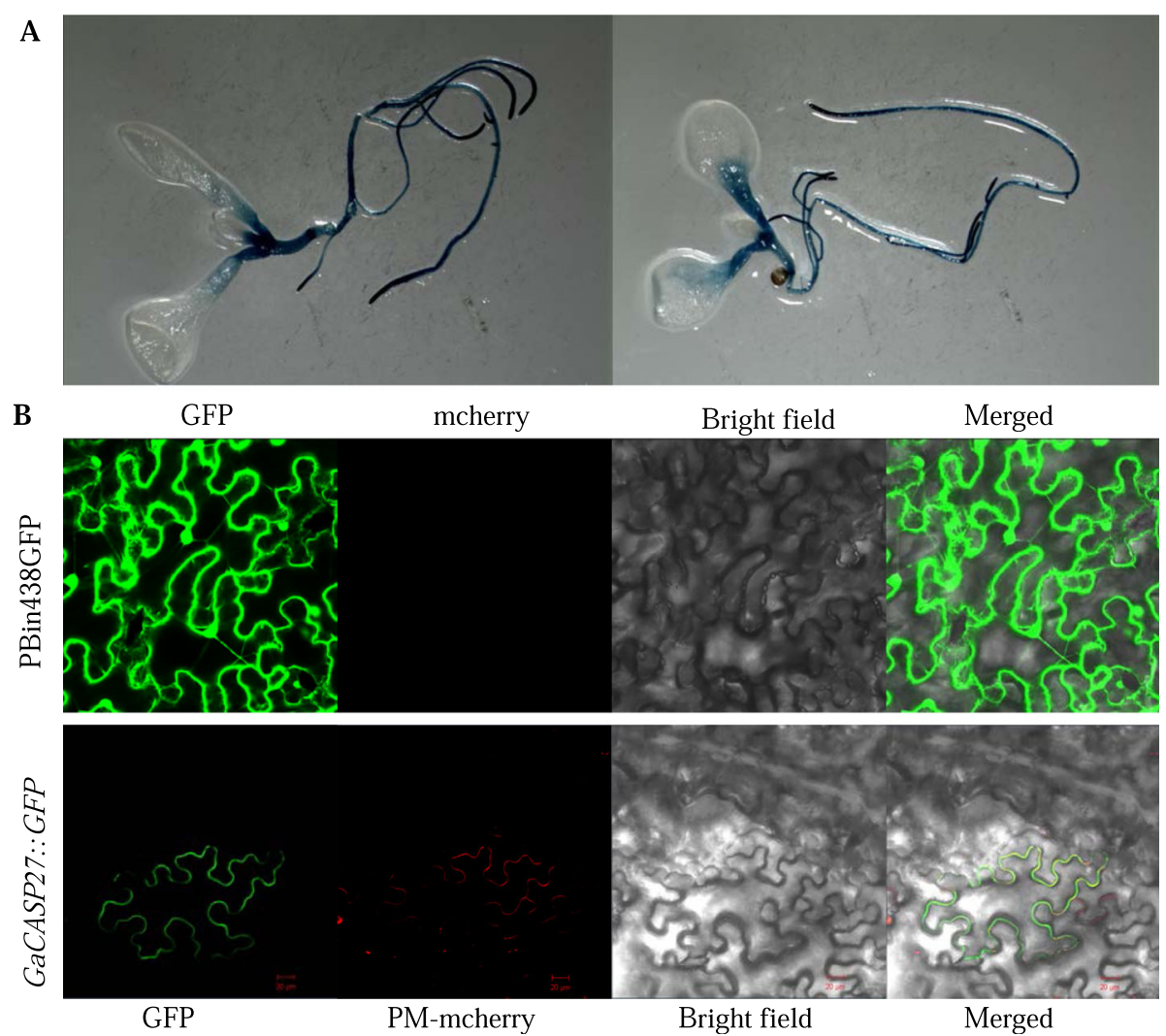

Fig. 5 The tissue and subcellular localization of GaCASP27. a GUS-staining analysis of the pGaCASP27::GUS transgenic Arabidopsis in primary roots. b Subcellular localization of GaCASP27 in N. benthamiana. GaCASP27::GFP signal was merged with that of the PM-mCherry (an intrinsic plasma membrane protein) marker in N. benthamiana. The vector of PBinGFP438 was used as the control. All experiments were analyzed with three biological repeats

under the control of CaMV35S promoter. The plasma membrane markers red fluorescent protein (RFP) and 35S-GaCASP27::GFP were co-transformed into the leaves of tobacco (Nicotiana benthamiana). Laser confocal microscopic results revealed that the empty control vector pBin438GFP presented a fluorescent signal in the cell membrane, nucleus and cytoplasm. By contrast, GaCASP27::GFP fusion proteins were co-localized with a plasma membrane marker in the plasma membrane, which confirmed our hypothesis (Fig. 5b). These results indicated that the GaCASP27 protein is a membrane protein.

\section{Silencing GaCASP27 increased the lateral root number}

The virus-induced gene-silencing (VIGS) method was used to further validate the functional role of GaCASP27 in root development. A $311 \mathrm{bp}$ fragment in the $3^{\prime}$ end of Ga08G0117 was isolated and cloned into pTRV1 to construct the TRV:GaCASP27 vector [27]. The empty vector TRV:00 was used as a negative control. After 2 weeks of Agrobacterium-based infiltration, the cotton plants harboring TRV:CLA1 showed photobleaching phenotype in young leaves, indicating that the VIGS experiment was successful (Fig. 6a). To further determine the gene silencing efficiency, qRT-PCR was used to evaluate the expression levels of GaCASP27 in both the TRV: GaCASP27 and TRV:00 control cotton plants. Compared with TRV:00 control plants, the expression level of GaCASP27 was drastically reduced in the TRV: GaCASP27 plants (Fig. 6b). The number of lateral roots in GaCASP27-silenced plants increased compared with the bank (CK) and negative control plants (Fig. 6c). The CASP genes Arabidopsis mutants Atcasp1/2/3 also exhibited a greater number of lateral roots compared with WT (Additional file 10). These findings suggest that the Casparian strip gene GaCASP27 negatively regulates the development of lateral roots in cotton and Arabidopsis.

\section{GaCASP27 was a target gene of GaMYB36}

Previous studies demonstrated that AtMYB36 could bind to the promoter of AtCASP via chromatin immunoprecipitation (ChIP)-qPCR [13]. The point mutation Atmyb36-1 had longer primary roots and misshaped lateral roots in early stages $[28,29]$. Our results also showed that Atmyb36-1 had null lateral roots (Additional file 10). In order to characterize the genetic 

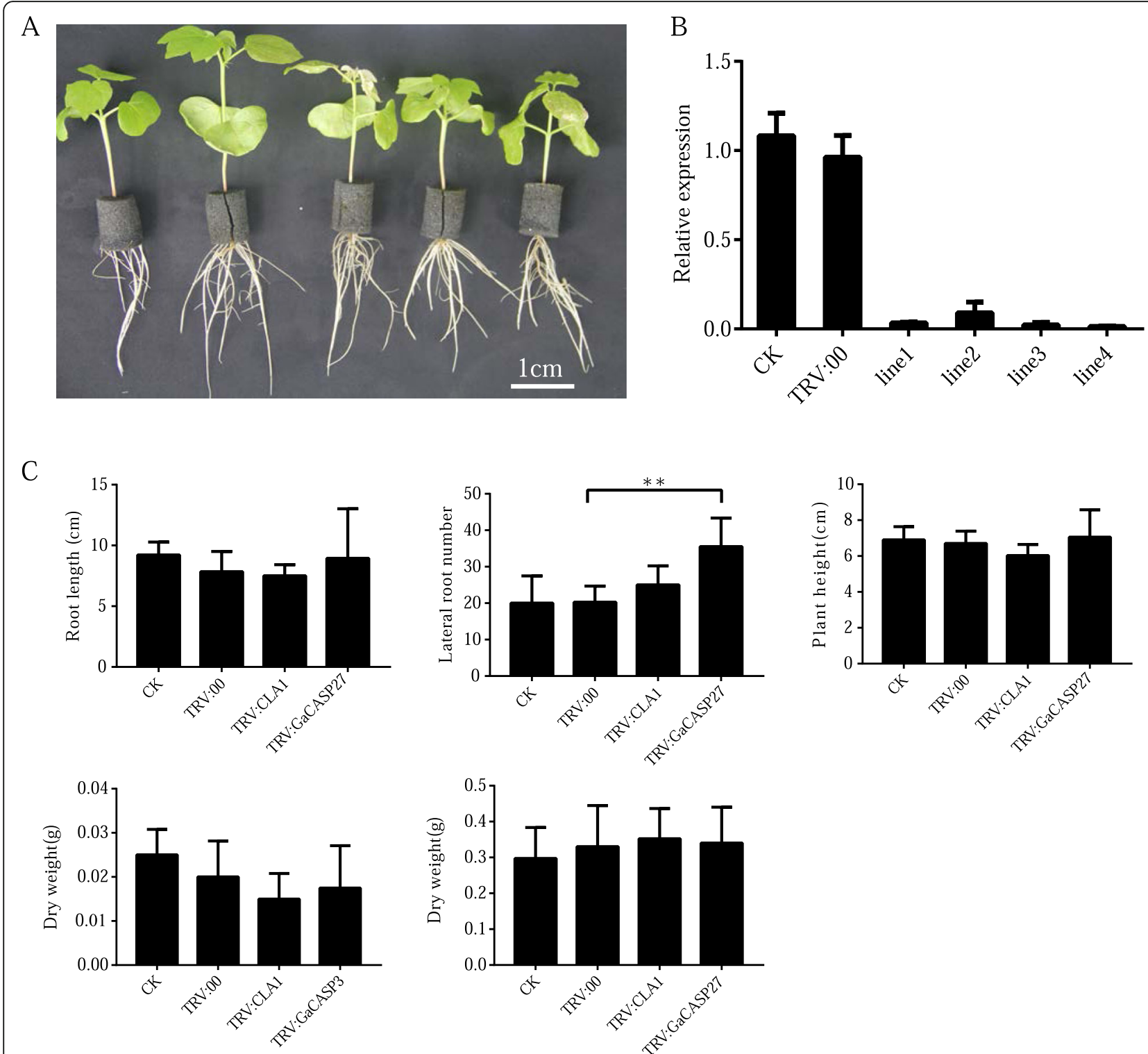

Fig. 6 GaCASP27 silenced plants have an increased number of lateral root phenotypes in G. arboreum. a Phenotypes of gene silencing plants were observed 2 weeks after infiltration. The plants from left to right were blank control (CK), negative control (TRV:00), positive control (TRV: CLA1) and two GaCASP27-silenced lines. b The expression levels of GaCASP27 in the blank control, negative control and silenced cotton plants were conducted through qRT-PCR All experiments were analyzed with three independent biological replicates. c Two weeks after infiltration, the root length, lateral root number and plant height were determined in GA0149 accession. All experiments were analyzed based on three independent biological replicates. The significant difference analysis was performed using the t-test at $P \leq 0.05$

interactions between MYB36 and GaCASP27, we analyzed the promoter element of GaCASP27 and identified seven MYB cis-elements MBSI CNGTT(A/G) (Fig. 7a and Additional file 7). We then performed a yeast onehybrid assay $(\mathrm{Y} 1 \mathrm{H})$. GaMYB36 was bound to the MBSI sequence of the GaCASP27 promoter and grew on SD medium containing the Leu/AbA (Fig. 7b). These results indicate that GaMYB36 plays an important role in regulating lateral root development by targeting GaCASP27.

\section{Discussion}

Characterization of Casparian gene family

Casparian strips are present in the roots of euphyllophytes, such as ferns and spermatophytes. They are involved in the defense against various biotic and abiotic stresses [28, 30-33]. Casparian strips also function as a receiver, hormone transporter, signal of calcium waves, respond to external environmental changes, and promote the growth and development of new lateral organs. 
A

-526 AATAAACTATTAAACTGAGCGGTTG TGAATTTTTTAAT pGaCASP27

-526 AATAAACTATTAAACTGAGCGCTTG TGAATTTTTTAAT pGaCASP27-mutant

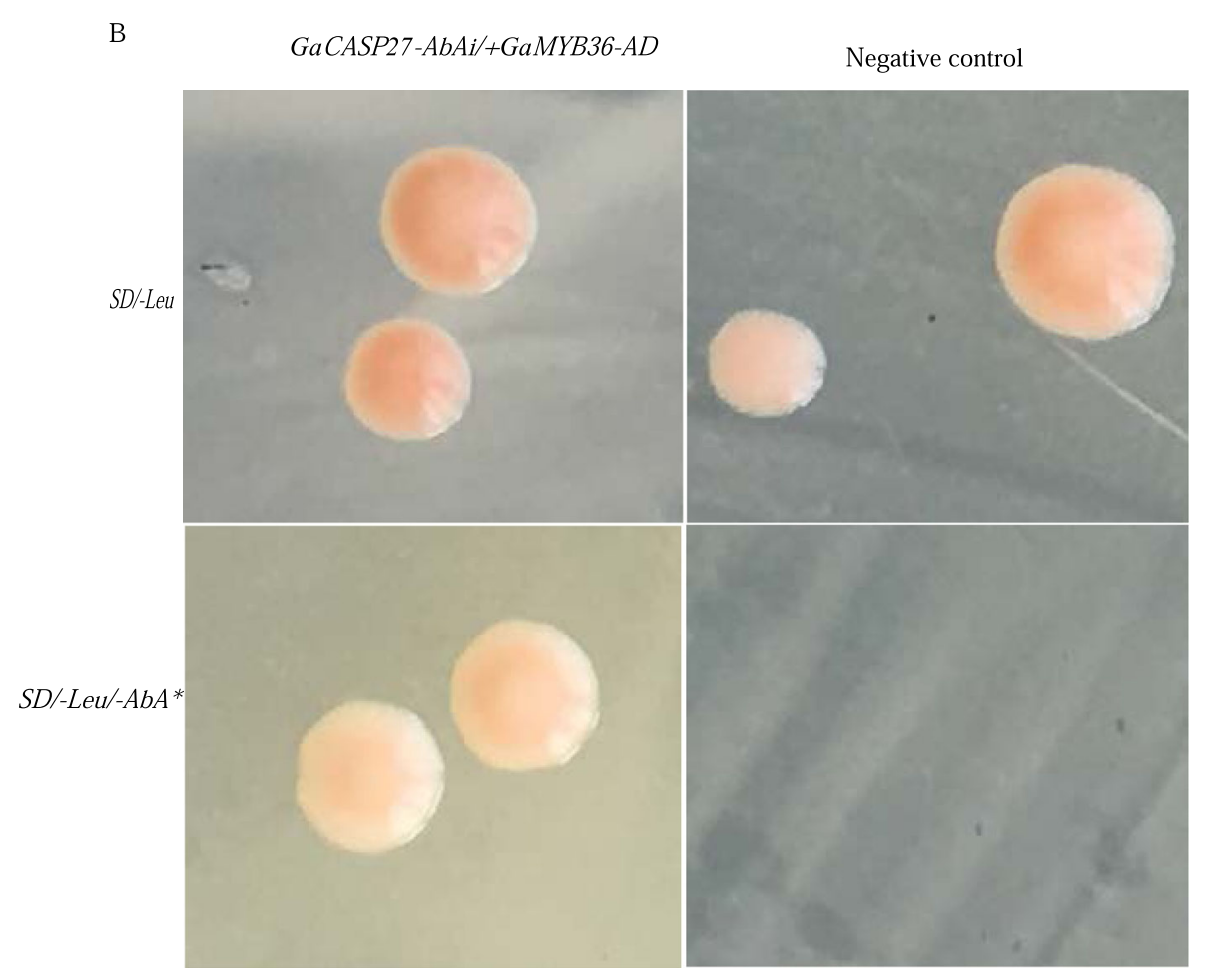

Fig. 7 GaMYB36 binds to the MBSI sequences of the GaCASP27 promoter in yeast. a The $2.0 \mathrm{~kb}$ GaCASP27 promoter region upstream of the ATG start codon includes predicted MBSI sequences (CGGTTG). $\mathbf{b}$ A yeast one-hybrid assay was performed to analyze the interaction between GaMYB36 and GaCASP27. Three biological repeats were performed

Proteins in the CASP family recruit a group of enzymes catalyzing lignin biosynthesis [34, 35]. However, the roles of CASP genes in the development of cotton roots have yet to be well studied.

In this study, we isolated 48, 91, 94 and 54 CASP genes from G. arboreum, G. barbadense, G. hirsutum and $G$. raimondii, respectively. Interestingly, the number of CASP genes in G. hirsutum was almost equal to that of G. raimondii and G. arboreum. This could be because the allotetraploid cotton genomes are composed of diploid A and D genome [36]. The number of CASP genes in cotton was more than that of Arabidopsis. The likely reason that cotton higher has more CASP genes is that it has a more complex genome. Tandem, whole-genome, and segmental duplications are the three major mechanisms contributing to the complexity of the genomic structure in higher plants over evolutionary time [37]. Gene duplicating is often related to forming of homologous genes in gene families [38]. Tandem duplication is usually caused by unequal crossings, while many of these occurrences can lead to the expansion or reduction of different gene family members [39]. Wang (2019) demonstrated that tandem replication within $A t$ and $D t$ subgenomes causes the expansion of the GhSOT gene family [40]. The WGD known as polyploidization, which results in double chromosomes and retained duplicates, is considered a primary driver of species diversification [41]. Wang (2018) found that WGD was the determining factor for the expansion of the GSK gene family [42]. Proximal duplication includes two gene copies that are closely located on the chromosome, but separated by a few genes [43]. Two adjacent gene duplications that originated from ancient tandem duplication events can be destroyed by inserting other genes, which is the source of proximal duplication [44]. Furthermore, the activity of localized transposon can lead to proximal duplication [45]. After performing a collinearity analysis, we identified the gene duplication events among CASP 
genes, and detected four tandem, eighteen whole-genome and two proximal duplication gene pairs in G. arboreum (Additional file 4). Dispersed duplicates create two gene copies that are neither neighboring to each other in the genome nor within paralogous chromosome segments [46]. Dispersed duplicates include transposable elements that are ubiquitous in the human genome, such as members of the Alu and $L 1$ family [47]. Stewart (2011) detected 1000 dispersed duplications using a database-free approach in the human genome [48]. The genome-wide analysis showed that 21 GaCASP genes were produced by dispersed duplication. This suggests that dispersed duplication could have contributed to the expansion of the GaCASP gene family in diploid G. arboreum ancestors.

Orthologs genes could share identical biological functions over evolutionary time [49]. In this study, 70, 39, and 45 orthologous $C A S P$ gene pairs were predicted between G. arboreum and G. hirsutum, G. arboreum and G. raimondii, and G. arboreum and G. barbadense, respectively (Additional file 5). CASPs from G. arboreum and Arabidopsis were divided into six subfamilies using a phylogenetic tree analysis. We observed that in the fourth group, five CASP genes (Ga08G0113, Ga08G0114, Ga08G0116, Ga08G0117, and Ga08G0118) shared close relationships with Arabidopsis AtCASP1, which regulated the development of lateral roots. Additionally, we aligned the protein sequences of GbCASPs, GaCASPs, GrCASPs and GhCASPs, and found that all of these CASPs contained four conserved transmembrane domains and two traces of active amino acids, suggesting that these proteins were highly conserved across species.

Gene structure determines gene function [50]. Both motif domain and analysis of gene structure demonstrated that CASP genes in the same subgroup shared a similar structure, suggesting that these CASP genes may have similar functions. The transcript analysis of GaCASP genes revealed that the same group of genes shared similar expression patterns in cotton. Previously published transcriptomic results revealed that CASP-like family genes are expressed in various tissues, with similar expression trends in the same group observed in Arabidopsis [51]. However, a higher root expression profile was reported for many genes in this family, including other CASP-like genes as well as $C A S P 1 / 2 / 3 / 4 / 5$ [51]. Our study identified five GaCASP genes, such as GaCASP24/25/26/27/28, that were specifically expressed in roots. This phenomenon prompted us to hypothesize that there is a relationship between GaCASP genes and root development.

\section{GaMYB36 involvement in root development by interacting directly with lateral root negative regulator GaCASP27}

MYB36 plays a critical role in the formation of Casparian strips by the localization of lignin polymerization in
Arabidopsis. MYB36 positively regulates cells, in Arabidopsis roots, from proliferation to differentiation [28]. The length of the primary root was significantly shorter in lines with MYB36 overexpression. However, the number of meristem cells increased in lines with the myb36 mutant [28]. In our study, we observed longer roots and less lateral root density in the Atmyb36 mutant plants, which is consistent with previous results (Additional file 10). According to Li (2018), MYB36 combines with the transcription factors SCARECROW (SCR) and SHORTROOT (SHR) to form a complex (SHR-SCR-MYB36), regulating the expression of CASPs and affecting Casparian strip formation [52]. However, in our study, MYB36 was directly bound to the promoter of GaCASP27 and positively regulated the expression of GaCASP27. Casparian strip formation plays a critical role in maintaining ROS homeostasis which negatively regulated lateral root development in Arabidopsis [53]. This was consistent with our study: the number of lateral roots increased in the GaCASP27 silenced plants. Furthermore, the single mutants Atcasp1, Atcasp 2 and Atcasp 3 possessed more lateral roots compared with WT (Additional file 10). This suggests that MYB36 is involved in root development by directly interacting with GaCASP 27 (which was mainly responsible for the formation of the Casparian strip) and negatively regulates the growth and development of lateral roots.

\section{Conclusions}

We identified 48, 54, 91, and 94 CASPs from G. arboreum, G. raimondii, G. barbadense, and G. hirsutum at the genome-wide level, respectively. Only 29 homologous GaCASPs gene pairs were detected in the four Gossypium species. WGD might be the primary reason for the expansion of CASPs in these four cotton species. Details about the on the phylogeny, gene structure, conserved motif composition, and physical positions of the chromosomes illustrated both the evolution and conservation of CASP genes. Most GaCASP genes were expressed across all of the different development stage of fiber, and root, stem and leaf tissues. However, five GaCASP24/25/26/27/28 genes were highly expressed in roots. Histochemical analysis and subcellular localization further demonstrated that the membrane protein GaCASP27 was also expressed in the roots. Additionally, a VIGS mediated knockdown analysis showed the GaCASP27 negatively regulated the development of lateral roots in cotton.

\section{Methods}

Identification and chromosomal distribution analysis of GaCASPs in Gossypium

The data of G. raimondii (JGI), G. arboreum (CRI), G. hirsutum (NAU) and G.barbadense (HAU) were downloaded 
from the CottonFGD (https://cottonfgd.org/about/down load.html), while the data of the protein sequence of Arabidopsis were downloaded from TAIR (https://www.arabi dopsis.org/). The hidden Markov model (HMM) seed file (Stockholm format) of the uncharacterized protein domain (PF04535) was downloaded from the Pfam website (http://pfam.xfam.org/) and used as a query when searching for Casparian strips (CASP) sequences using the HMMER software (http://hmmer.org). The conserved, uncharacterized protein domains were confirmed by the SMART (http://smart.emblheidelberg.de/), Pfam (http:// pfam.xfam.org/), and NCBI-CDD databases. The biophysical properties of the CASP proteins were obtained from CottonFGD (https://cottonfgd.org/about/download.html). Information about the chromosomal position of the GaCASPs genes was obtained from CottonFGD (https:// cottonfgd.org/analyze/), while the "GaCASPs distribution map" was drawn by MapChart [54].

\section{Gene collinearity and duplication analysis}

The TBtools software (https://github.com/CJ-Chen/TBtools) was used to analyze collinearity pairs of $C A S P$ genes from G. arboreum and three other Gossypium species. MCScanX (http://chibba.pgml.uga.edu/mcscan2/) was used to analyze gene duplication events in G. arboreum genomes [55]. Tandem duplication events were identified when two consecutive genes were distributed on the same chromosomes under the threshold of $\mathrm{e}<10^{-20}$. Segmental duplications were defined as duplication events within large chromosomal regions in the same chromosom $[37,56]$. Segmentally duplicated pairs among CASP proteins from $G$. arboreum and the three other Gossypium accessions were analyzed using TBtools (https://github.com/CJ-Chen/TBtools). Tandem duplications were identified using a BLAST-Like Alignment Tool (BLAT) [57].

\section{Characterization and sequence analysis of related CASP genes}

The genomic and coding sequences of the CASP genes were downloaded from TAIR (https://www.arabidopsis. org/) and cottonFGD (https://cottonfgd.org/) and analyzed using the Gene Structure Display Server online software (GSDS2.0) (http://gsds.cbi.pku.edu.cn/) to predict gene structures. Multi-protein sequence alignment of the CASPs was conducted by the DNAMAN software, while the phylogenetic tree was built using the MEGA 7 software with the maximum likelihood (ML) and JTT + G models [58-61]. We performed 1000 bootstrap replications to produce bootstrap values. The identification of the transmembrane region for GaCASPs was predicted by the TMHMM program [51]. The protein sequences of GaCASPs were submitted to the MEME online software (http://meme-suite.org/tools/meme) to predict the conserved motif.

\section{Plant materials and seedling growth}

The varieties of diploid Asiatic cotton (G. arboreum L.) wildtype DPL971(GA0146) and fuzzless mutant DPL972 (GA0149) were introduced from Yunnan province, China, before 1998 and kept in the Chinese National Germplasm Mid-term Genebank (Anyang, China) [62]. In our present study, these two varieties were provided by Mid-term Genebank. The roots of GA0149 and GA0146 were harvested from seedlings at two- and four-old-day development stages, while the fresh leaves and stems were harvested from three-week-old seedlings grown in the green house. Samples of fiber and ovules were collected in the morning at $-2,0,+3,+5$ and +8 day post anthesis (DPA) from cotton grown under field conditions. All of the samples were frozen in liquid nitrogen and stored at $-80^{\circ} \mathrm{C}$ for RNA-Seq and gene expression analysis. We isolated each sample with at least 3-5 g from a mixture of the tissue from three individuals. Each sample includes three independent biological experiments and three technical replicates.

$N$. benthamiana seedlings were grown under $16 \mathrm{~h}$ light/ $8 \mathrm{~h}$ dark cycles, with temperatures of $25^{\circ} \mathrm{C}$ during the day and $23^{\circ} \mathrm{C}$ at night, for subcellular location. For the tissue location, Arabidopsis (Columbia) seedlings were grown in MS (Murashige and Skoog) medium at $20^{\circ} \mathrm{C}$ for 7 days and subsequently transferred to nutrient soil.

\section{RNA sequencing and analysis}

Total RNA was extracted from harvested cotton tissues using the RNAprep Pure Plant Kit (TIANGEN, Beijing, China), and examined by a NanoDrop-Photometer ${ }^{\circ}$ spectrophotometer RNA. For the next RNA-sequencing, a total of $5 \mu \mathrm{g}$ RNA was sent to Biomarker company (Beijing). Each sample was analyzed with three biological replicates. The sequencing protocol and analysis were performed according to the reference instructions, and were modified slightly [63]. Simply, the enriched RNA was broken into $200 \mathrm{nt}$ RNA short fragments., after which the first and second-cDNA were synthesized. The synthesized double-stranded cDNA fragments were subjected to end-repair/dA-tail and ligation adapters. The suitable fragments were then purified by agarose gel electrophoresis and enriched by PCR amplification. Finally, the cDNA library was constructed using the Illumina HiSeq 2500 sequencing platform. The raw data were aligned to the standard line GA0024 genome using Tophat2 software [64]. The gene expression level was calculated using RPKM (reads per kilobase of exon model per million fragments mapped) through the Cufflinks software [65]. The differential expressed genes were defined by the following parameters: FDR $<0.05$ and $\mid \log 2$ Ratio $\mid \geq$ 1 . The gene ID and its sample information used in this 
study are listed in Additional file 6. The heat map was generated using the TBtools software.

\section{Plasmid construction and plant transformation}

The up-stream 2000 bp promoter fragment of GaCASP27 (Ga08G0117) was amplified from GA0149 genomic DNA with specific primers. The PCR fragment was subcloned into pMD19-simple T vectors for sequencing, and the correct promoter sequence was submitted to PlantCARE (http://bioinformatics.psb.ugent.be/webtools/plantcare/ $\mathrm{html} /$ ) for Cis-acting elements prediction [66]. The GaCASP27 (Ga08G0117) promoter fragment was digested using ScaI/XbaI, inserted into the ScaI/XbaI sites of the PBI121 vector and replaced the $35 \mathrm{~S}$ promoter. As a result, $p$ GaCASP27:: GUS recombinant vector was generated, and transformed into the Agrobacterium tumefaciens strain GV3101. The floral dipping method was used for the Arabidopsis transformation [67].

The GFP reporter vector GaCASP27::GFP was constructed according to the following: the full-length coding sequence (CDS) of GaCASP27 (Ga08G0117) without termination codons, was amplified from GA0149 cDNA using the special primers. The PCR fragment was then subcloned into a pMD19-simple $\mathrm{T}$ vector and digested using the single enzyme Sall. The digested fragment cloned into linearized the pBinGFP438 vector was digested with the same enzyme to construct the plasmid GaCASP27::GFP, which was driven by the $35 \mathrm{~S}$ promoter [68]. The recombinant plasmid GaCASP27::GFP was transferred into the A. tumefaciens strain LBA4404 by liquid nitrogen freezing and thawing.

\section{GUS histochemical staining assays and subcellular location} The homozygous $p$ GaCASP27::GUS Arabidopsis positive plants were selected for tissue analysis. Seeds were grown on MS medium with $0.7 \%$ agar powder. After 2 weeks, the seedlings were soaked in a GUS staining solution containing $1 \mathrm{mM} \mathrm{X}$-Gluc, $100 \mathrm{mM}$ sodium phosphate ( $\mathrm{pH} 7.0)$, $10 \mathrm{mM}$ EDTA, $0.5 \mathrm{mM}$ potassium ferricyanide, $0.5 \mathrm{mM}$ potassium ferrocyanide and $0.3 \%(\mathrm{v} / \mathrm{v})$ Triton X-100. After staining at $37^{\circ}$ Covernight, the tissues were decolorized using a series of different concentration gradients alcohol [69]. The samples were then photographed using optical microscopy with a CCD camera (Leica Microsystems, Germany). The GUS histochemical staining assays were performed with three biological replicates.

Strains of A. tumefaciens LBA4404, carrying the GaCASP27::GFP recombinant plasmid and the control pBinGFP438, were cultured overnight in an LB liquid medium containing $50 \mu \mathrm{g} / \mathrm{mL}$ kanamycin, $30 \mu \mathrm{g} / \mathrm{mL}$ rifampicin and $50 \mu \mathrm{g} / \mathrm{mL}$ streptomycin at $28^{\circ} \mathrm{C}, 200 \mathrm{rpm}$. When the $\mathrm{OD}_{600}$ reached $0.8-1.0$, the $A$. tumefaciens cells were centrifuged and suspended with infiltration buffer $\left(10 \mathrm{mM} \mathrm{MgCl}_{2}, 10 \mathrm{mM}\right.$ MES, pH 5.7, $200 \mu \mathrm{M}$ acetosyringone). The suspension was then incubated at room temperature for $2 \mathrm{~h}$. The recombinant plasmid and the control pBinGFP438 A. tumefaciens cells were infiltrated into 4 weeks old tobacco leaves by a $1 \mathrm{~mL}$ plastic syringe. The plants were cultured overnight in a dark environment and transferred to a chamber for 2 days with a $16 \mathrm{~h} / 8 \mathrm{~h}$ photoperiod. The fluorescence signal in transfected tobacco leaves was observed using confocal laser scanning microscopy (Zeiss LSM510 META, Germany). Subcellular location was performed with three biological replicates.

\section{Virus-induced gene silencing of GaCASP3 in tissues}

A. tumefaciens-mediated VIGS was performed in the seedlings of GA0149. The $311 \mathrm{bp}$ fragment of Ga08G0117 was cloned into the $\mathrm{XbaI} / \mathrm{SacI}$ sites of the pYL156 vector to generate the TRV:GaCASP27 recombinant plasmid. The TRV:GaCASP27 vector was transferred into the A. tumefaciens strain GV3101. Seeds of GA0149 cultivars were sterilized and sown in the sand to germination. After 3 days, the seedlings were transferred into plastic containers with a half-strength of Hoagland nutrient solution. When the seedlings cotyledon had completely spread, they were used for VIGS assays. The A. tumefaciens strain LBA4404 carrying TRV2 (helper vector), TRV1 (empty vector), TRV: CLA1 (positive control) and TRV: GaCASP27 plasmids were grown overnight in LB containing rifampicin $(30 \mu \mathrm{g} /$ $\mathrm{mL})$ and kanamycin $(50 \mu \mathrm{g} / \mathrm{mL})$ at $28^{\circ} \mathrm{C}, 200 \mathrm{rpm}$. When the $\mathrm{OD}_{600}$ value of the culture reached $1.3-1.5$, the $A$. tumefaciens cells were centrifuged and suspended in an isopyknic infiltration medium containing $10 \mathrm{mM} \mathrm{MgCl}_{2}, 10$ $\mathrm{mM}$ 2-(4-Morpholino) ethane sulfonic acid (MES) and $200 \mu \mathrm{M}$ acetosyringone. After incubating for $3 \mathrm{~h}$ at $25^{\circ} \mathrm{C}$, the $A$. tumefaciens carrying TRV1, TRV: CLA1 and TRV: GaCASP3 was mixed with A. tumefaciens harboring TRV2 at a ratio of 1:1, respectively. The $A$. tumefaciens solutions were infiltrated into the two fully expanded cotyledons of the GA0149 plants via a needleless syringe. Untreated (CK) and empty vector (TRV: 00) transformed plants were used as experimental controls. The Cloroplastos alterados 1 (CLA1) gene was used as a positive control [70]. These infiltrated plants were grown in the greenhouse at $25^{\circ} \mathrm{C}$.

Approximately 2 weeks after infiltration, the true leaves of GA0149 were collected for RNA isolation and qRT-PCR analysis. cDNA was synthesized using a PrimeScript ${ }^{\text {tem }}$ RT reagent kit with gDNA Eraser (TaKaRa, Japan). The quantitative real-time (qRT)-PCR was performed using SYBR Premix Ex Taq (TaKaRa, Japan) in a 7500 Fast Real-Time PCR system (Applied Biosystems, Inc., California USA). The Histon 3 gene was used as a reference. The relative expression levels of the GaCASP27 gene were calculated using the $2^{-\triangle \Delta C t}$ method [71]. The experiment of virusinduced gene silencing of GaCASP27 in cotton was performed with at least three replicates. 


\section{Assay of biomass}

For assessing the biomass, 2 weeks after infiltration, the fresh seedlings of CK, TRV: 00, TRV: CLA1 and TRV: GaCASP3 were weighed using the analytical balance at the laboratory bench at room temperature. For measuring dry weight, the enzymes of the fresh seedlings were deactivated in an oven at $100-105^{\circ} \mathrm{C}$ for $10 \mathrm{~min}$, and then dried to a constant weight at $70^{\circ} \mathrm{C}$, after which the seedling dry weight was obtained using an analytical balance. These experiments were conducted with three biological replicates and three technical repeats.

\section{Analysis of root and hypocotyl traits}

The lengths of the primary roots and the hypocotyls were measured using a ruler. The number of root hairs of the 15-day-old seedlings were counted using an electron microscope, and the number of lateral roots of the 15day-old seedlings was counted using a hand magnifier. The phenotype of each root was captured using a Canon color CCD camera (EOS M5), while the color of the root was photographed using an Olympus CKX53 microscope fitted with an attached CCD camera imaging system. At least 10 lines of CK, TRV: 00, TRV: CLA1 and TRV: GaCASP3 plants were recorded for each repeat.

\section{Yeast one-hybrid assay}

The yeast one-hybrid assay was performed according to the Matchmaker One-Hybrid System User Manual (Clontech). The 3xMYB binding elements of GaCASP27, attached with HindIII and SalI, were synthesized according to the primer synthesis method. The forward primers and reverse primers were mixed in a ratio of 1:1 to form a double-stranded DNA. The double-stranded DNA was cloned into the HindIII/SalI sites of pAbAi vector to generate the bait recombinant plasmid. The full length of GaMYB36 was amplified from GA0149 cDNA using the special primers, and was then inserted into pGADT7 (Clontech) to create the prey plasmid. The bait plasmid was transferred into Y1HGold strains to obtain yeast strains with bait vector, while the prey plasmid was transferred into Y1HGold which contained bait vector according to the LiAc transformation method [72]. The interactions were selected on SD/-Leu plates containing $100 \mathrm{ng} / \mathrm{ml}$ Aureobasidin A (AbA), which were growth in the chamber for $3-5$ days at $30^{\circ} \mathrm{C}$.

All of the gene-specific primers of the amplifications or vector constructions, as well as qRT-PCR analysis, are listed in Additional file 1.

\section{Statistical analysis}

All of the experiments were performed with at least three replicates. Data were subjected to statistical analysis using one-way ANOVA, while Duncan's multiple range test was used to assess the means $\pm \mathrm{SD}$.

\section{Supplementary information}

Supplementary information accompanies this paper at https://doi.org/10. 1186/s12864-020-6723-9.

Additional file 1: Table S1. All primers used in this study.

Additional file 2: Table S2. The information of CASPS gene family in cotton.

Additional file 3: Table S3. The general information of the GaCASP family.

Additional file 4: Table S4. The duplication CASP gene pairs in cotton.

Additional file 5: Table S5. Orthologous relationships between $G$. arboreum and other three Gossypium species.

Additional file 6: Table S6. The transcript expression profiles of the GaCASP family in GA0146 and GA0149.

Additional file 7: Table S7. Tissue specificity regulatory elements found in the GaCASP27 promoter region.

Additional file 8: Figure S1. Chromosomal location map analysis of CASP genes in G. arboreum.

Additional file 9: Figure S2. pGaGASP27::GUS positive seedlings were screened in the Kanamycin/Cephalosporin MS medium. The red arrow indicates positive transgenic seedlings.

Additional file 10: Figure S3. Phenotypes of wild type, Atmyb36, Atcasp1, Atcasp2 and Atcasp3 under normal growth condition. (A) Lateral root phenotypes of WT and mutants. The seedlings were grown in vertically solid MS medium for 6 days, and transferred into MS medium for continued 7 days. (B) The number of the lateral roots was counted after 15 days transferring to MS. Three biological replicates were performed. The significant difference analysis was performed using oneway ANOVA and Tukey's HSD test $(P<0.05)$.

\section{Abbreviations}

CASPs: Casparian stirp strip membrane domain proteins; $A$.

thaliana: Arabidopsis thaliana; A. tumefaciens: Agrobacterium tumefaciens; VIGS: Virus-Induced Gene-Silencing; RPKM: Reads Per Kilobase of exon model per Million fragments mapped; CDS: Coding Sequence; GFP: Green Fluorescent Protein; GSDS: Gene structure display server; WGD: Whole genome duplication; Y1H: Yeast One-Hybrid assay

\section{Acknowledgements}

We thank Prof. Philip N. Benfey (Howard Hughes Medical Institute, Duke University, Durham) for providing the Arabidopsis mutants myb36-1. We also thank Muhammad Yasir for kindly revising this manuscript.

\section{Authors' contributions}

XYW performed most of the experiments, bioinformatics analysis and participated in writing the manuscript. YMZ and LYW analyzed the transcriptome data and draw the circle graph. ZEP and SPH helped to cultivate all the plant materials and conduct qRT-PCR. QG and BJC performed yeast one-hybrid assays. XMD and WFG conceived the project and designed the research. All authors read and approved the manuscript.

\section{Funding}

This work was supported by funding from the National Key Technology R\&D Program, the Ministry of Science and Technology (2016YFD0100203, 2016 YFD0100306 and 2017YFD0101601). The funders had no role in study design, data collection and interpretation, or the decision to submit the work for publication.

\section{Availability of data and materials}

All related data are available within the manuscript and its additional files. The RNA sequences raw data were deposited in the Biological Research Project Data (BioProject), National Center for Biotechnology Information (NCBI) under the accession numbers PRJNA622455.

Ethics approval and consent to participate Not applicable. 


\section{Consent for publication}

Not applicable.

\section{Competing interests}

The authors declare that they have no competing interests.

\section{Author details}

${ }^{1}$ State Key Laboratory of Cotton Biology, Institute of Cotton Research, Chinese Academy of Agricultural Sciences, Anyang 455000, Henan, China. ${ }^{2}$ Crop Information Center, College of Plant Science and Technology, Huazhong Agricultural University, Wuhan 430070, China. ${ }^{3}$ Key Laboratory of Cultivation and Protection for Non-Wood Forest Trees, Ministry of Education, Central South University of Forestry and Technology, Ministry of Education, Changsha 410004, China.

Received: 19 July 2019 Accepted: 6 April 2020

Published online: 04 May 2020

\section{References}

1. Peret B, De Rybel B, Casimiro I, Benkova E, Swarup R, Laplaze L, Beeckman T, Bennett MJ. Arabidopsis lateral root development: an emerging story. Trends Plant Sci. 2009;14(7):399-408.

2. Hochholdinger F, Park WJ, Sauer M, Woll K. From weeds to crops: genetic analysis of root development in cereals. Trends Plant Sci. 2004;9(1):42-8.

3. Osmont KS, Sibout R, Hardtke CS. Hidden branches: developments in root system architecture. Annu Rev Plant Biol. 2007;58(58):93-113.

4. Enstone DE, Peterson CA, Ma F. Root endodermis and exodermis: structure, function, and responses to the environment. J Plant Growth Regul. 2003; 21(4):335-51.

5. Geldner N. The endodermis. Annu Rev Plant Biol. 2013;64(64):531-58.

6. Nakayama T, Shinohara H, Tanaka M, Baba K, Ohnishi MO, Matsubayashi Y. A peptide hormone required for casparian strip diffusion barrier formation in arabidopsis roots. Science. 2017;355:284-6.

7. Roppolo D, De Rybel B, Denervaud Tendon V, Pfister A, Alassimone J, Vermeer JE, Yamazaki M, Stierhof YD, Beeckman T, Geldner N. A novel protein family mediates casparian strip formation in the endodermis. Nature. 2011;473(7347):380-3.

8. Naseer S, Lee Y, Lapierre C, Franke R, Nawrath C, Geldner N. Casparian strip diffusion barrier in Arabidopsis is made of a lignin polymer without suberin. Proc Natl Acad Sci U S A. 2012;109(25):10101-6.

9. Lee $\mathrm{Y}$, Rubio MC, Alassimone J, Geldner N. A mechanism for localized lignin deposition in the endodermis. Cell. 2013;153(2):402-12.

10. Barbosa ICR, Murcia NR, Geldner N. The Casparian strip-one ring to bring cell biology to lignification? Curr Opin Biotechnol. 2019;56:121-9.

11. Pfister A, Barberon M, Alassimone J, Kalmbach L, Lee $Y$, Vermeer JE, Yamazaki M, Li GW, Maurel C, Takano J, Kamiya T, Salt DE, Roppolo D, Geldner N. A receptor-like kinase mutant with absent endodermal diffusion barrier displays selective nutrient homeostasis defects. Elife. 2014;3:e03115.

12. Alassimone J, Fujita S, Doblas VG, Dop MV, Barberon M, Kalmbach L, Vermeer JEM, Murcia NR, Santuari L, Hardtke CS, Geldner N. Polarly localized kinase SGN1 is required for Casparian strip integrity and positioning. Nature Plants. 2016;2(8):1-10.

13. Kamiya T, Borghi M, Wang P, Danku JM, Kalmbach L, Hosmani PS, Naseer S, Fujiwara T, Geldner N, Salt DE. The MYB36 transcription factor orchestrates Casparian strip formation. Proc Natl Acad Sci U S A. 2015;112(33):10533-8.

14. Zhang HB, Li Y, Wang BH, Chee PW. Recent advances in cotton genomics. Int J Plant Genomics. 2008;2008:742304.

15. Rengel Z, Damon PM. Crops and genotypes differ in efficiency of potassium uptake and use. Physiol Plant. 2008;133(4):624-36.

16. Zhang Z, Yang FQ, Li B, Eneji EA, Li JM, Duan LS, Wang BM, Li ZH, Tian XL. Coronatine-induced lateral-root formation in cotton (Gossypium hirsutum) seedlings under potassium-sufficient and -deficient conditions in relation to auxin. J Plant Nutr Soil Sci. 2009;172(3):435-44.

17. Kharkina TG, Ottosen CO, Rosenqvist E. Effects of root restriction on the growth and physiology of cucumber plants. Physiol Plant. 2010;105(3):434-41.

18. Meng Z, Zhaohong M, Rui Z, Chengzhen L, Jianmin W, Yanling W, Honghong Z, Sandui G, Xianlong Z. Expression of the Rice Arginase gene OsARG in cotton influences the morphology and nitrogen transition of seedlings. PLoS One. 2015;10(11):e0141530.
19. Wang Y, Meng Z, Liang C, Meng Z, Wang Y, Sun G, Zhu T, Cai Y, Guo S, Zhang $R$, Lin Y. Increased lateral root formation by CRISPR/Cas9-mediated editing of arginase genes in cotton. Sci China Life Sci. 2017;60(5):524-7.

20. Kundu A, Das S, Basu S, Kobayashi Y, Kobayashi Y, Koyama H, Ganesan M, Whelan J. GhSTOP1 a C2H2 type zinc finger transcription factor is essential for aluminum and proton stress tolerance and lateral root initiation in cotton. Plant Biol. 2018;21(1):35-44.

21. Du X, Huang G, He S, Yang Z, Sun G, Ma X, Li N, Zhang X, Sun J, Liu M. Resequencing of 243 diploid cotton accessions based on an updated a genome identifies the genetic basis of key agronomic traits. Nat Genet. 2018;50(6):796-802.

22. Finn RD, Bateman A, Clements J, Coggill P, Eberhardt RY, Eddy SR, Heger A, Hetherington K, Holm L, Mistry J, Sonnhammer ELL, Tate J, Punta M. Pfam: the protein families database. Nucleic Acids Res. 2014;42(Database issue): D222-30.

23. Berntsson RP, Smits SH, Schmitt L, Slotboom DJ, Poolman B. A structural classification of substrate-binding proteins. FEBS Lett. 2010;584(12):2606-17.

24. Wang W, Cheng YY, Chen DD, Liu D, Hu MJ, Dong J. The catalase gene family in cotton: genome-wide characterization and bioinformatics analysis. Cells. 2019;8:86.

25. Chen Q, Chen QJ, Sun GQ, Zheng K, Yao ZP, Han YH. Genome-wide identification of cyclophilin gene family in cotton and expression analysis of the fibre development in Gossypium barbadense. Int J Mol Sci. 2019;20:349.

26. Holub EB. The arms race is ancient history in Arabidopsis, the wildflower. Nat Rev Genet. 2001;2(7):561-27.

27. Qu J, Ye J, Geng YF, Sun YW, Gao SQ, Zhang BP, Chen W, Chua NH. Dissecting functions of KATANIN and WRINKLED1 in cotton fiber development by virusinduced gene silencing. Plant Physiol. 2012;160(2):738-48.

28. Liberman LM, Sparks EE, Moreno-Risueno MA, Petricka JJ, Benfey PN. MYB36 regulates the transition from proliferation to differentiation in the Arabidopsis root. Proc Natl Acad Sci U S A. 2015;112(39):12099-104.

29. Fernandez-Marcos M, Desvoyes B, Manzano C, Liberman LM, Benfey PN, Del Pozo JC, Gutierrez C. Control of Arabidopsis lateral root primordium boundaries by MYB36. New Phytol. 2017;213(1):105-12.

30. Wu X, Lin J, Lin Q, Wang J, Schreiber L. Casparian strips in needles are more solute permeable than endodermal transport barriers in roots of Pinus bungeana. Plant Cell Physiol. 2005;46(11):1799-808.

31. Yoon J, Choi $\mathrm{H}, \mathrm{An} \mathrm{G}$. Roles of lignin biosynthesis and regulatory genes in plant development. J Integr Plant Biol. 2015;57(11):902-12.

32. Liska D, Martinka M, Kohanova J, Lux A. Asymmetrical development of root endodermis and exodermis in reaction to abiotic stresses. Ann Bot. 2016; 118:667-74

33. Le Roy J, Blervacq AS, Creach A, Huss B, Hawkins S, Neutelings G. Spatial regulation of monolignol biosynthesis and laccase genes control developmental and stress-related lignin in flax. BMC Plant Biol. 2017;17(1):124.

34. Roppolo D, Boeckmann B, Pfister A, Boutet E, Rubio MC, DTendon DV, Vermeer JE, Gheyselinck J, Xenarios I, Geldner N. Functional and evolutionary analysis of the casparian strip membrane domain protein family. Plant Physiol. 2014;165(4):1709-22.

35. Hosmani PS, Kamiya T, Danku J, Naseer S, Geldner N, Guerinot ML, Salt DE. Dirigent domain-containing protein is part of the machinery required for formation of the lignin-based Casparian strip in the root. Proc Natl Acad Sci U S A. 2013;110(35):14498-503.

36. Li F, Fan G, Lu C, Xiao G, Zou C, Kohel RJ, Ma Z, Shang H, Ma X, Wu JY, Liang XM, Huang G. Genome sequence of cultivated upland cotton (Gossypium hirsutum TM-1) provides insights into genome evolution. Nat Biotechnol. 2015;33(5):524-30.

37. Cannon SB, Mitra A, Baumgarten A, Young ND, May G. The roles of segmental and tandem gene duplication in the evolution of large gene families in Arabidopsis thaliana. BMC Plant Biol. 2004;4(1):1-21.

38. Zhang J. Evolution by gene duplication: an update. Trens Ecol Evol. 2003;18:292-8,

39. Achaz G, Coissac E, Viari A, Netter P. Analysis of intrachromosomal duplications in yeast Saccharomyces cerevisiae: a possible model for their origin. Mol Biol Evol. 2000;17(8):1268-75.

40. Wang LY, Liu XY, Wang XY, Pan ZE, Geng XL, Chen BJ. Identification and characterization analysis of sulfotransferases (SOTS) gene family in cotton (Gossypium) and its involvement in fiber development. BMC Plant Biol. 2019; 19:595-608.

41. Ren R, Wang HF, Guo CC, Zhang N, Zeng LP, Chen YM. Widespread whole genome duplications contribute to genome complexity and species diversity in Angiosperms. Mol Plant. 2018;11:414-28. 
42. Wang LL, Yang ZE, Zhang B, Yu DQ, Liu J, Gong Q. Genome-wide characterization and phylogenetic analysis of GSK gene family in three species of cotton: evidence for a role of some GSKs in fiber development and responses to stress. BMC Plant Biol. 2018;18:330-51.

43. Wang YP, Wang XY, Paterson AH. Genome and gene duplications and gene expression divergence: a view from plants. J Ann N Y Acad Sci. 2012; 1256(1):1-14.

44. Freeling M. Bias in plant gene content following different sorts of duplication: tandem, whole-genome, segmental, or by transposition. Annu Rev Plant Biol. 2009;60:433-53.

45. Zhao XP, Si Y, Hanson RE, Crane CF, Price HJ, Stelly DM, Wendel JF, Paterson $\mathrm{AH}$. Dispersed repetitive DNA has spread to new genomes since polyploid formation in cotton. Genome Res. 1998;8(5):479-92.

46. Ganko EW, Meyers BC, Vision TJ. Divergence in expression between duplicated genes in Arabidopsis. Mol Biol Evol. 2007;24(10):2298-309.

47. Kroon M, Lameijer EW, Lakenberg N, Hehir-Kwa JY, Thung DT, Slagboom PE. Detecting dispersed duplications in high-throughput sequencing data using a database-free approach. Bioinformatics. 2016;32(4):505-10.

48. Stewart C, Kural D, Stromberg MP, Walker JA, Konkel MK, Stutz AM. A comprehensive map of mobile element insertion polymorphisms in humans. PLoS Genet. 2011;7:e1002236.

49. Altenhoff AMDC, Eisen JA. Phylogenetic and functional assessment of Orthologs inference projects and methods. PLoS Comput Biol. 2009;5(1): e1000262.

50. Shaul O. How introns enhance gene expression. Int J Biochem Cell Biol. 2017:91:145-55. S1357272517301541.

51. Yang J, Ding C, Xu B, Chen C, Narsai R, Whelan J, Hu Z, Zhang M. A Casparian strip domain-like gene, CASPL, negatively alters growth and cold tolerance. Sci Rep. 2015;5(14299):1-11.

52. Li P, Yu Q, Gu X, Xu C, Qi S, Wang H, Zhong F, Baskin TI, Rahman A, Wu S. Construction of a functional casparian strip in non-endodermal lineages is orchestrated by two parallel signaling systems in Arabidopsis thaliana. Curr Biol. 2018;28(17):2777-86 e2772.

53. Li N, Sun L, Zhang L, Song Y, Hu P, Li C, Hao FS. AtrbohD and AtrbohF negatively regulate lateral root development by changing the localized accumulation of superoxide in primary roots of Arabidopsis. Planta. 2015; 241(3):591-602.

54. Voorrips RE. MapChart: software for the graphical presentation of linkage maps and QTLs. J Hered. 2002;93(1):77-8.

55. Wang $Y$, Tang $H$, Debarry JD, Tan $X$, Li J, Wang $X$, Lee TH, Jin H, Marler $B$ Guo H, et al. MCScanX: a toolkit for detection and evolutionary analysis of gene synteny and collinearity. Nucleic Acids Res. 2012;40(7):e49.

56. Li Q, Yu H, Cao PB, Fawal N, Mathé C, Azar S, Wang HC. Explosive tandem and segmental duplications of multigenic families in Eucalyptus grandis. Genome Biol Evol. 2015;7(4):1068-81.

57. Doerks T, Copley RR, Schultz J, Ponting CP, Bork P. Systematic identification of novel protein domain families associated with nuclear functions. Genome Res. 2002;12(1):47-56.

58. Thompson JD, Gibson TJ, Plewniak F, Jeanmougin F, Higgins DG. The CLUSTAL_X windows interface: flexible strategies for multiple sequence alignment aided by quality analysis tools. Nucleic Acids Res. 1997;25(24): 4876-82.

59. Stéphane Guindon DJF, Lefort V. New algorithms and methods to estimate maximum-likelihood phylogenies: assessing the performance of PhyML 3.0. Syst Biol. 2010;59(3):307-21.

60. Darriba D, Taboada GL, Doallo R, Posada D. ProtTest 3: fast selection of bestfit models of protein evolution. Bioinformatics. 2011;27(8):1164-5.

61. Kumar S, Stecher G, Peterson D, Tamura K. MEGA-CC: computing core of molecular evolutionary genetics analysis program for automated and iterative data analysis. Bioinformatics. 2012;28(20):2685-6.

62. Wang S, Zhao GH, Jia YH, Du XM. Cloning and characterization of a CAP gene expressed in Gossypium arboreum Fuzzless mutant. Crop Sci. 2008; 48(6):2314-20

63. Shu C, Zhao M, Anderson JP, Garg G, Singh KB, Zheng W, Wang C, Yang M, Zhou E. Transcriptome analysis reveals molecular mechanisms of sclerotial development in the rice sheath blight pathogen Rhizoctonia solani AG1-IA. Funct Integr Genomics. 2019;19:743-58.

64. Trapnell C, Pachter L, Salzberg SL. TopHat: discovering splice junctions with RNA-Seq. Bioinformatics. 2009;25(9):1105-11.

65. Mortazavi A, Williams BA, McCue K, Schaeffer L, Wold B. Mapping and quantifying mammalian transcriptomes by RNA-Seq. Nat Methods. 2008;5(7):621-8.
66. Lescot M. PlantCARE, a database of plant cis-acting regulatory elements and a portal to tools for in silico analysis of promoter sequences. Nucleic Acids Res. 2002;30(1):325-7.

67. Clough SJ, Bent AF. Floral dip: a simplified method for Agrobacteriummediated transformation of Arabidopsis thaliana. Plant J. 1998;16(6):735-43.

68. Liu TL, Song TQ, Zhang X, Yuan HB, Su LM, Li WL, Xu J, Liu SH, Chen LL, Chen TZ, Zhang MX, Gu LC, Zhang BL, Dou DL. Unconventionally secreted effectors of two filamentous pathogens target plant salicylate biosynthesis. Nat Commun. 2014;5:4686

69. Jefferson RA, Kavanagh TA, Bevan MW. GUS fusions: ,B-glucuronidase as a sensitive and versatile gene fusion marker in higher plants. EMBO J. 1987; 6(13):3901-7.

70. Gao X, Britt RC Jr, Shan L, He P. Agrobacterium-mediated virus-induced gene silencing assay in cotton. J Vis Exp. 2011;54:1-4.

71. Livak KJ, Schmittgen TD. Analysis of relative gene expression data using realtime quantitative PCR and the $2^{-\Delta \Delta C}$, method. Methods. 2001;25(4): 402-8.

72. Gietz RD, Schiestl RH, Willems AR, Woods RA. Studies on the transformation of intact yeast cells by the LiAc/SS-DNA/PEG procedure. Yeast. 1995;11(4): 355-60.

\section{Publisher's Note}

Springer Nature remains neutral with regard to jurisdictional claims in published maps and institutional affiliations.
Ready to submit your research? Choose BMC and benefit from:

- fast, convenient online submission

- thorough peer review by experienced researchers in your field

- rapid publication on acceptance

- support for research data, including large and complex data types

- gold Open Access which fosters wider collaboration and increased citations

- maximum visibility for your research: over $100 \mathrm{M}$ website views per year

At BMC, research is always in progress.

Learn more biomedcentral.com/submissions 